\title{
Nasofrontal Outflow Tract Visibility in Computed Tomography Imaging of Frontal Sinus Fractures
}

\author{
Kevin Bush, BSc, MD, FRCS(C) ${ }^{1}$ Menno Huikeshoven, MD, $\mathrm{PhD}^{1}$ Nathan Wong, $\mathrm{BSc}^{1}$ \\ ${ }^{1}$ Department of Plastic Surgery, University of British Columbia, \\ Vancouver, British Columbia, Canada \\ Craniomaxillofac Trauma Reconstruction 2013;6:237-240

\begin{abstract}
Address for correspondence Kevin Bush, BSc, MD, FRCS(C), Department of Plastic Surgery, University of British Columbia, \#8081200 Burrard St., Vancouver, British Columbia V6Z 2C7, Canada
\end{abstract} \\ (e-mail: kevinlbush@gmail.com).
}

\begin{abstract}
\section{Keywords}

- CT scan

- frontal sinus fracture

- nasofrontal duct

- nasofrontal outflow tract

The choice of frontal sinus fracture treatment is based on multiple factors, one of which is injury to the nasofrontal outflow tract (NFOT). Computed tomography (CT) imaging of the NFOT can play an important role in the decision process. We sought to assess the visibility of the NFOT on CT scans in frontal sinus fractures. Patients with frontal sinus fractures (including the posterior table) receiving a CT scan from April 1st 2001 to December 31st 2009 were included. Scans were retrospectively assessed for available views (axial, coronal, and sagittal), slice thickness, inclusion of the anatomical NFOT region in the scanned area, and visibility of the NFOT. A total of 170 patients were included. In majority $(71 \%)$ of patients NFOT was visible on one or more views, whereas in $33 \%(N=56)$ of patients had three complete views (complete anatomical NFOT region scanned in three views). In this subgroup, the ability to assess the NFOT increased to $89 \%$. When selecting patients with three complete views of $\leq 2 \mathrm{~mm}$ slice thickness ( $N=47$ ), the ability to assess the NFOT increased to $96 \%$. In conclusion, when assessing the NFOT using CT imaging, having three complete views (axial, coronal, and sagittal) and $\mathrm{a} \leq 2 \mathrm{~mm}$ slice thickness greatly increases the NFOT visibility.
\end{abstract}

Frontal sinus fractures make up approximately 5 to $15 \%$ of craniomaxillofacial fractures. ${ }^{1-3}$ They are typically the result of high-energy injuries, are commonly associated with other facial fractures, and can lead to significant complications such as meningitis, encephalitis, intracranial abscesses, osteomyelitis, and mucoceles. ${ }^{4-6}$ Multiple factors play a role in the choice between surgical or nonsurgical treatment of frontal sinus fractures and subsequently may influence the risk of complications. These factors include fracture type, presence of comminution, cerebrospinal fluid leakage, extent of posterior table involvement, neurological status of the patient, preference of the treating physician and (suspicion of) nasofrontal outflow tract (NFOT) injury. Computed tomography (CT) imaging has long been an indispensable tool in evaluating several of these factors. With regards to the NFOT, CT assessment of injury can however be difficult. Recently, diagnostic criteria have been described in an attempt to identify specific findings on CT imaging that carry a high suspicion for injury and/or obstruction of the NFOT. ${ }^{4,7}$ These criteria include fracture of the frontal sinus floor, fracture of the medial aspect of the anterior table (anterior ethmoid cells), and frank bony outflow tract obstruction. When attempting to apply the suggested criteria to our own patient population, we found significant inconsistencies in visualization and visibility of the NFOT in patients diagnosed with frontal sinus fractures due to variations in CT scan parameters. This study was therefore designed to retrospectively assess several CT parameters in patients diagnosed with frontal sinus fractures in our institution between 2001 and 2009 and correlate these to visibility of the frontal sinus and the NFOT.

\section{Methods}

This study was a retrospective chart and imaging review of patients admitted to Vancouver General Hospital and received

December 17, 2012

accepted after revision

February 11, 2013

published online

September 24, 2013
Copyright $\odot 2013$ by Thieme Medical Publishers, Inc., 333 Seventh Avenue, New York, NY 10001, USA. Tel: +1(212) 584-4662.
DOI http://dx.doi.org/ 10.1055/s-0033-1349214. ISSN 1943-3875. 
diagnosed with frontal sinus fractures including the posterior wall and was approved by University of British Columbia's ethics. Patients admitted and diagnosed between April 1st 2001 and December 31st 2009 were identified as follows. The following ICD-10 (International Classification of Diseases) codes that could potentially include or represent frontal sinus fractures were used to search the hospital database: S02.0, "fracture of vault of skull;" S02.1, "fracture of base of skull;" S02.7, "multiple fractures skull and facial bones;" S02.8, "fractures of other skull and facial bones;" and S02.9, "fractures of skull and facial bones, part unspecified." Using this database, radiology reports around each patient's admission date were reviewed for possible frontal sinus fractures. For the resulting patients, (preoperative) CT scans were reviewed for presence of posterior wall fractures. Patients with only hardcopy CT scans were excluded. CT scans meeting inclusion criteria were retrospectively reviewed for visualization and visibility of the frontal sinus and the NFOT. Visualization was assessed using the following parameters: availability of axial, coronal and/or sagittal views; inclusion of the complete anatomical region of the frontal sinus and the NFOT in the scanned section; and slice thickness in millimeters. Visibility was scored by assessing whether the NFOT could be identified on the scan. All scans were reviewed by K.B. and M.H.

\section{Results}

A total of 1507 patients were identified using the described ICD codes. After reviewing radiology reports, 219 out of these 1507 were identified as having frontal sinus fractures. After reviewing CT images, 178 patients were determined to have fractures including the posterior wall. In eight patients, a digital CT scan was not available (only hardcopy) and they were subsequently excluded. This resulted in a total of 170 patients included for review.

Of 170 scans, 169 (99\%) included axial views, 118 (69\%) coronal views, 64 (38\%) sagittal views, and 62 (36\%) all three views. Of the 62 scans where all three views were available, 56 (33\% of total) included the complete anatomical region of the frontal sinus and the NFOT in the scanned region. These were termed "complete" scans. Slice thickness in all views ranged from 0.8 to $7 \mathrm{~mm}$ (mean $1.7 \mathrm{~mm}$ (SD 1.0), median 1.3).

As presented in - Table 1, the NFOT was visible in $71 \%$ $(N=120)$ of the total of 170 scans. When selecting the subgroup of "complete" scans $(N=56)$, the visibility of the NFOT increased to $89 \%(N=50)$. Finally, when selecting a subgroup of "complete" scans with a slice thickness of $\leq 2 \mathrm{~mm}$ $(N=47)$, the visibility of the NFOT increased to $96 \%(N=45)$.

\section{Discussion}

The assessment of NFOT injury in frontal sinus fractures can be a challenging task. Osseous and/or soft tissue injury may lead to obstruction of the tract with subsequent decrease or complete blocking of frontal sinus drainage. This may increase the risk of short- and long-term complications observed after frontal sinus fractures. When the posterior wall of the frontal sinus is included in the fracture, obstruction of the NFOT may lead to ascending intracranial infections such as meningitis, encephalitis, abscesses, and osteomyelitis.

Furthermore, a chronically obstructed NFOT may lead to mucoceles, an infrequent but serious complication. CT diagnostic criteria for suspicion of NFOT injury have previously been identified as frontal sinus floor fracture, fracture of the medial wall of the anterior table (anterior ethmoid cells), and frank bony obstruction of the outflow tract. ${ }^{8-10}$ Recently, Rodriguez et $\mathrm{al}^{4}$ and Stanwix et $\mathrm{al}^{7}$ correlated these CT criteria to their experience with 1097 frontal sinus fracture patients over a 26-year period and concluded that the radiological diagnosis of NFOT injury in frontal sinus fractures plays a decisive role in surgical planning.

Proper assessment of these criteria may be dependent on the technical quality of the CT scan. As this may require standardization of CT scan protocols in suspected frontal sinus fracture patients we sought to assess the technical quality of CT scans of patients diagnosed with frontal sinus fractures.

The results of our study indicate that the visibility of the NFOT on CT scans increases when a combination of axial, coronal, and sagittal views are available, the complete anatomical region of the frontal sinus and the NFOT is included in the scanned area, and the slice thickness is $2 \mathrm{~mm}$ or less. The visibility of the NFOT increased from $71 \%$ in the total group of included patients diagnosed with frontal sinus fractures to $96 \%$ in the subgroup that met these three criteria. Optimizing the visibility of the NFOT will benefit the assessment of the earlier mentioned criteria for NFOT injury on CT imaging.

Several comments could be considered with regards to the results and implications of this study. First, the available views, slice thickness, and scanned area may not be the only technical aspects that play a role in the visibility of the NFOT as it could also be influenced by other factors such as the presence of artifacts or differences in scanning protocols. Second, although beyond the scope of this study, soft tissue injury to the NFOT on CT imaging is difficult to assess unless air can be seen throughout the entire tract. Injury to the mucosal lining of the NFOT may still lead to significant

Table 1 Visibility of the nasofrontal outflow tract on computed tomography scans made for frontal sinus fractures

\begin{tabular}{|l|l|l|l|}
\hline & $\begin{array}{l}\text { All scans } \\
(N=170)\end{array}$ & $\begin{array}{l}\text { Scans with three "complete views" } \\
(N=56)\end{array}$ & $\begin{array}{l}\text { Scans with three "complete views" } \\
\text { and slice thickness } \leq 2 \text { mm } \\
(N=47)\end{array}$ \\
\hline NFOT visible on scan $(N)$ & $71 \%(120)$ & $89 \%(50)$ & $96 \%(45)$ \\
\hline
\end{tabular}

Abbreviation: NFOT, nasofrontal outflow tract.

Note: "Complete views," axial, coronal, and sagittal, all including the complete anatomical region of the frontal sinus and the nasofrontal outflow tract. 
scarring and subsequent obstruction despite the possible absence of the three earlier described (bony) criteria. Last, it should be noted that there currently is no clinical correlation that confirms the radiographic findings of NFOT injury. Correlation between X-ray findings and techniques, such as sinus endoscopy, could be useful in allowing better interpretation of the CT scan findings and determining the clinical value of the criteria described in this study.

In conclusion, this study indicates that meeting several technical criteria in CT imaging can increase NFOT visibility in frontal sinus fractures. We therefore recommend that in patients with a clinical suspicion of a frontal sinus fracture, CT imaging should include three views (axial, coronal, and sagittal) of the entire anatomical region of the frontal sinus and the NFOT with a slice thickness of $2 \mathrm{~mm}$ or smaller. Meeting these criteria will aid the assessment of injury to the NFOT and subsequently the decision process for appropriate treatment.

\section{References}

1 Rohrich RJ, Hollier LH. Management of frontal sinus fractures. Changing concepts. Clin Plast Surg 1992;19(1):219-232
2 Lakhani RS, Shibuya TY, Mathog RH, Marks SC, Burgio DL, Yoo GH. Titanium mesh repair of the severely comminuted frontal sinus fracture. Arch Otolaryngol Head Neck Surg 2001;127(6):665-669

3 Gerbino G, Roccia F, Benech A, Caldarelli C. Analysis of 158 frontal sinus fractures: current surgical management and complications. J Craniomaxillofac Surg 2000;28(3):133-139

4 Rodriguez ED, Stanwix MG, Nam AJ, et al. Twenty-six-year experience treating frontal sinus fractures: a novel algorithm based on anatomical fracture pattern and failure of conventional techniques. Plast Reconstr Surg 2008;122(6):1850-1866

5 Chen KT, Chen CT, Mardini S, Tsay PK, Chen YR. Frontal sinus fractures: a treatment algorithm and assessment of outcomes based on 78 clinical cases. Plast Reconstr Surg 2006;118(2): 457-468

6 Manolidis S, Hollier LH Jr. Management of frontal sinus fractures. Plast Reconstr Surg 2007;120(7, Suppl 2):32S-48S

7 Stanwix MG, Nam AJ, Manson PN, Mirvis S, Rodriguez ED. Critical computed tomographic diagnostic criteria for frontal sinus fractures. J Oral Maxillofac Surg 2010;68(11):2714-2722

8 Harris L, Marano GD, McCorkle D. Nasofrontal duct: CT in frontal sinus trauma. Radiology 1987;165(1):195-198

9 Heller EM, Jacobs JB, Holliday RA. Evaluation of the frontonasal duct in frontal sinus fractures. Head Neck 1989;11(1):46-50

10 Landsberg R, Friedman M. A computer-assisted anatomical study of the nasofrontal region. Laryngoscope 2001;111(12):2125-2130 